\title{
EVALUACIÓN DE CALIDAD DE CHAPAS FOLIADAS DE CEREZO, NOGAL Y ALISOS PROCEDENTE DE TROZAS DE RALEO DE PLANTACIONES JÓVENES
}

\author{
Loewe, Verónica ${ }^{3}$; Fuchslocher, Francisco ${ }^{4}$; Delard, Claudia ${ }^{5}$ y del Río, Rodrigo ${ }^{6}$
}

\section{RESUMEN}

Plantaciones jóvenes destinadas a la producción de madera de alto valor fueron raleadas y parte del producto del raleo, trozas con diámetros mayores a $20 \mathrm{~cm}$, fue procesada a fin de determinar la factibilidad de valorizar esta madera mediante el uso más preciado, chapas decorativas obtenidas mediante foliado.

Las mayores limitantes para la obtención de chapas de calidad fueron los reducidos diámetros de las trozas y la presencia de nudos, lo que indica que este material debe orientarse a otros usos, así como la necesidad de aplicar un manejo adecuado y oportuno para reducir la presencia de defectos. Además, ante su inexistencia, se propone una escala de clasificación de calidad de chapas decorativas foliadas.

El foliado de maderas de las especies analizadas se justifica con trozas de calidad y de mayores diámetros, las que pueden obtenerse mediante técnicas como la arboricultura, a fin de maximizar el valor del material producido.

Se recomienda continuar realizando evaluaciones de foliado con trozas de mayores diámetros de raleos posteriores, así como evaluar otros usos para material de raleos intermedios a fin de identificar aplicaciones que permitan su valorización.

Palabras clave: Madera de alto valor, Chapas foliadas

\section{SUMMARY}

Young planted forests oriented to high value timber production were thinned and part of the obtained product, logs with a diameter over $20 \mathrm{~cm}$, were processed to determine the feasibility of obtaining the most valued product, veneers derived from slicing.

Main limiting factors to obtain high quality veneers were the reduced log diameters and the knots presence, indicating that this material should be oriented to other uses, as well as the need of an appropriated and opportune management to reduce defects presence. Furthermore, given the inexistence of a quality veneer classification, a standard is proposed.

Slicing of the studied species timber is justified when processing high quality and high diameter logs, which can be obtained through techniques such as arboriculture, in order to maximize the produced material value.

Further log slicing evaluations are recommended with superior diameter logs from future commercial thinning, as well as to identify other uses for small diameter logs utilization.

Keywords: High value timber, Slicing veneers

\footnotetext{
${ }^{3}$ Ingeniero Forestal, Jefe de Proyectos, Instituto Forestal, Chile. vloewe@infor.cl

${ }^{4}$ Ingeniero Forestal, Gerente General de Foliadora de Maderas Gorbea (FOLIMAG).

${ }^{5}$ Ingeniero Forestal, Investigador, Instituto Forestal, Chile.

${ }^{6}$ Ingeniero Agrónomo, Investigador, Instituto Forestal, Chile.
} 


\section{INTRODUCCIÓN}

En las últimas décadas en Europa se ha verificado un aumento del interés por el establecimiento de plantaciones de latifoliadas de alto valor (Kerr y Evans, 1993; Gabriel et al., 2005) debido a la disponibilidad de terrenos, a las limitaciones para importar maderas valiosas tropicales y a su elevado precio de mercado. Es por ello que cobró relevancia la arboricultura de calidad (Regione Piemonte, 2001; Minotta, 2003), que busca obtener el máximo volumen de madera de la mejor calidad posible de cada árbol en particular.

Para ello se han propuesto numerosas especies, entre las cuales se encuentran nogal común (Juglans regia L.) y cerezo común (Prunus avium L.) como especies principales, y alisos (Alnus glutinosa, A. cordata) (Ente Nazionale per la Cellulosa e per la Carta, 1990; Montero et al., 2003; Loewe et al., 2013), que también se han empleado como especies acompañantes que favorecen el desarrollo de las primeras (Binkley, 2003; Buresti y Frattegiani, 1994).

La calidad de la madera se define como la aptitud del material para satisfacer determinadas exigencias o usos específicos (Borghini y Massafra, 2002), con sus propias reglas y clasificaciones en cada caso, y por ello no se la puede definir en términos absolutos sin considerar el uso y las técnicas de transformación.

Los parámetros considerados en la evaluación de la calidad de la madera incluyen aspectos dimensionales y cualitativos. Los primeros corresponden a las dimensiones de la troza (diámetro y largo). Entre los cualitativos están todos los atributos y defectos presentes en la madera a raíz de su origen biológico, incluyendo forma, coloraciones anómalas (como por ejemplo el veteado verde en cerezo común) o alternancia de colores marcada (Figura $\mathrm{N}^{\circ} 1$ ), fibra torcida, madera de tensión/reacción, pudriciones, nudos (diámetro, ubicación y cantidad) y tipo (sano, no sano, muerto, con pudrición, con corteza incluida), heridas, entre otros. Cabe señalar que los nudos sanos generan mellas en los cuchillos y pérdidas de material foliado, por lo que, afectan el rendimiento (de $\mathrm{m}^{2}$ de chapa por $\mathrm{m}^{3}$ de troza).

Obviamente, mientras mayor sea la calidad de las trozas y por consiguiente con un uso potencial más preciado, mayor es el precio de las mismas, 1,5 a 6 veces entre trozas foliables y aserrables, y de hasta 9 veces en el caso de nogal (Wiedenbeck et al., 2004), lo que constituye un incentivo para aplicar un manejo que promueva un mayor rendimiento de trozas foliables. El precio de ellas no solo depende de la calidad, sino que también de la especie, del mercado y de la estrategia de abastecimiento y distribución de la empresa elaboradora.

En particular, se consideran de calidad para foliado, que es el uso más preciado existente, trozas de dimensiones superiores a $3 \mathrm{~m}$ de largo y a $30 \mathrm{~cm}$ de diámetro, rectas, y atractivas, parámetro subjetivo que depende del color, veteado y defectos (Wiedenbeck et al., 2004). Se excluyen para este uso aquellas que presenten los siguientes defectos: pudrición, nudos vivos o muertos, grietas por heladas, orificios de insectos, separación entre anillos, madera de reacción, grietas a modo de estrella, médula excéntrica, alteración del color, ovalización, inclusión de corteza, curvaturas, acanaladuras y fibra helicoidal.

Algunos defectos permitidos con limitaciones corresponden a ahusamiento, restos de corteza e inclusión de resinas o gomas (Berti et al., 2003). Idealmente las trozas deben presentar un ritmo de crecimiento anual constante, es decir, con anillos homogéneos, lo que determina su comportamiento en el tiempo, ya que permite que mantengan inalteradas sus características y no se rajen.

Existen normas para varios usos de la madera, incluyendo trozas (EN 1316-1), madera aserrada (CEN/TS 12169:2008), madera laminada (CEN/TS 13307-2:2009), embalajes (EN 12246:1999), pisos de madera (CEN/TS 13810-2:2003), tableros (EN 14915:2013+A1:2017), y otros. No obstante, en el caso de las chapas foliadas, el uso más valioso que se da a la madera, dadas las particularidades de las maderas de diferentes especies y la dificultad de hacer una evaluación objetiva de la calidad (se trata siempre de una evaluación subjetiva que suele ser controvertida), no existen normas que describan la calidad de chapas foliadas (Berti, 2017, com. personal). 


\section{OBJETIVOS}

Los objetivos de este trabajo fueron evaluar color, textura y apariencia, y la factibilidad de foliado de trozas procedentes de raleos de plantaciones jóvenes (16 a 21 años de edad), manejadas según principios de la arboricultura, orientadas a la producción de madera de calidad, a fin de determinar posibles usos que permitan valorizar el material producido durante la rotación.

\section{MATERIAL Y METODO}

\section{Material}

Durante los meses de junio y julio del año 2017 se efectuaron raleos en tres plantaciones experimentales. Algunas de las trozas obtenidas se seleccionaron para su posterior foliado ejecutado en Folimag (www.folimag.cl), empresa especializada en la elaboración de chapas decorativas (foliadas). Fueron procesadas en total 23 trozas; 2 de cerezo común (Prunus avium), 17 de alisos (Alnus cordata, A. glutinosa) y 4 de nogal común (Juglans regia), con un diámetro medio de $23 \mathrm{~cm}$ y $1,6 \mathrm{~m}$ de largo. El Aliso no es una especie comercial en el mercado de chapas, a diferencia del nogal y cerezo, que son requeridos constantemente, pero no obstante ello se consideró interesante evaluar su calidad y aptitud para este fin.

Las características técnicas de las trozas no cumplían con los estándares de la industria para producir chapas foliadas decorativas. En particular, para obtener chapas decorativas interesantes para el mercado de láminas decorativas se necesita cumplir con un promedio de ancho de la chapa terminada de 15 a $17 \mathrm{~cm}$, lo que con trozas delgadas como las evaluadas es prácticamente imposible.

\section{Método}

La secuencia de trabajo fue la siguiente:

Foliado: Dados los diámetros de las trozas solo se prepararon dos caras de foliado y este se realizó en un Foliadora Cremona TN 4.000, a un espesor de 0,58 mm.

Secado de Chapas: Se realizó en un secador Babcock a $135^{\circ} \mathrm{C}$, obteniéndose chapas con un contenido de humedad del $12 \%$.

\section{Guillotinado.}

Clasificación: Dada la inexistencia de clasificación estética de chapas foliadas, se elaboró una clasificación considerando las recomendaciones del Dr. Stefano Berti, especialista italiano de renombre mundial en maderas de alto valor, quien es Presidente del Comité Italiano de Normas de la Madera, y representante de dicho país ante la Unión Europea (CEN) en estas materias.

El trabajo con el Dr. Berti se realizó en la sede del Istituto per la Valorizzazione del Legno e delle Specie Arboree (IVALSA) del Consiglio Nazionale delle Ricerche (CNR), ubicada en Sesto Fiorentino, Italia.

La escala propuesta para clasificar chapas foliadas considera las siguientes categorías:

Clase A: Color homogéneo, sin ningún defecto.

Clase B: Presencia de algunos defectos como color heterogéneo y nudos sanos (Figuras $\mathrm{N}^{\circ} 1$ y $\mathrm{N}^{\circ} 2$ ), excluyendo defectos de mayor impacto, como pudriciones, nudos muertos, etc. Se aceptan nudos pequeños (diámetro máximo de $3 \mathrm{~cm}$ ) y brotes epicórmicos (diámetro máximo del grupo de $5 \mathrm{~cm}$ ) (Figura $\mathrm{N}^{\circ} 3$ ), en cantidad limitada (máximo total de 15 y 10 , respectivamente en cada chapa) idealmente en las secciones terminales de las chapas. 
Clase C: Presencia de defectos de mayor impacto, tales como pudriciones, nudos muertos o con inclusión de corteza (Figuras $\mathrm{N}^{\circ} 4$ y $\mathrm{N}^{\circ} 5$ ), así como con nudos sanos que por número o dimensión exceden lo aceptable en clase $\mathrm{B}$.

Clase D: Descarte por elevada cantidad de defectos graves, tales como presencia de médula, nudos muertos, pudriciones, etc., que afectan más del $60 \%$ de la superficie de la chapa.

Esta escala de clasificación se aplicó a cada paquete de chapas recibido de la empresa foliadora. Remanentes del proceso se presentan en la Figura $N^{\circ} 6$. La empresa, por su parte, también aplicó la clasificación que emplea a nivel industrial para las chapas que produce.

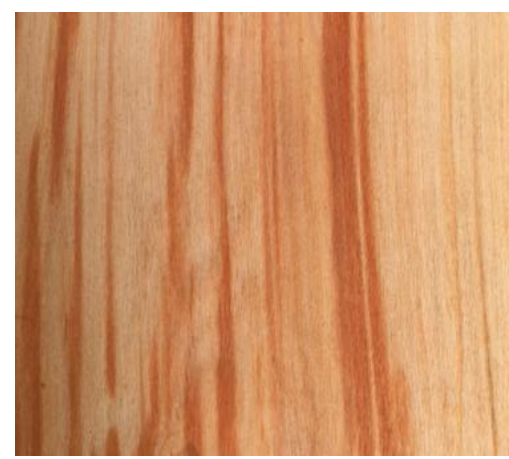

Figura $\mathrm{N}^{\circ} 1$

COLOR HETEROGÉNEO CONTRASTANTE
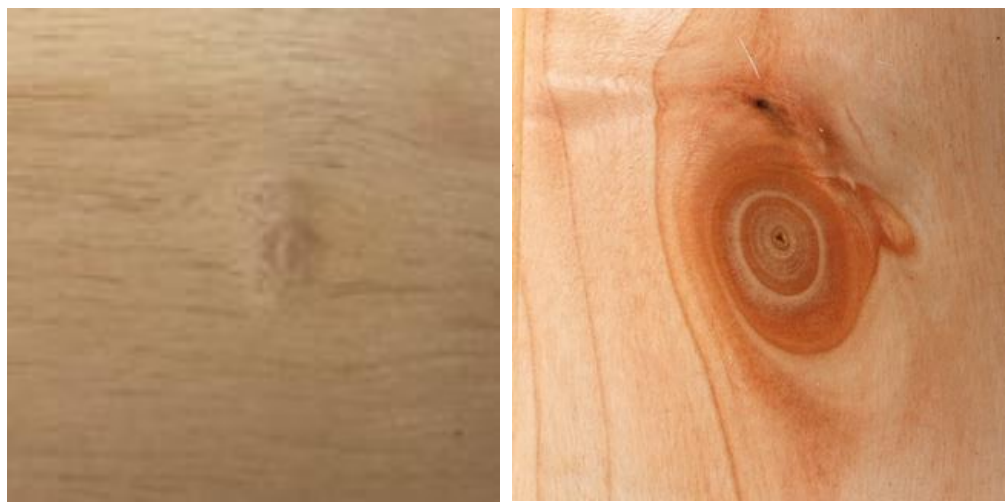

Figura $\mathbf{N}^{\circ} 2$

NUDOS SANOS 


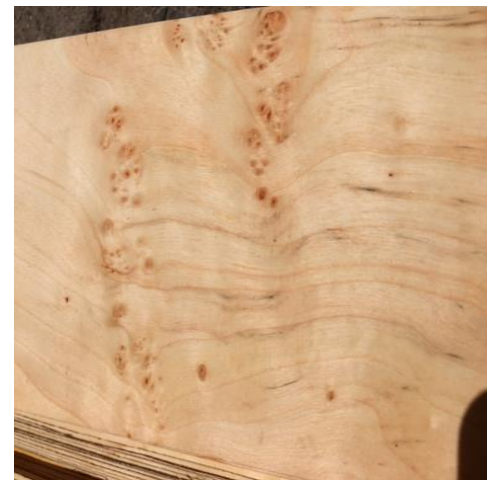

Figura $\mathrm{N}^{\circ} 3$ BROTES EPICÓRMICOS
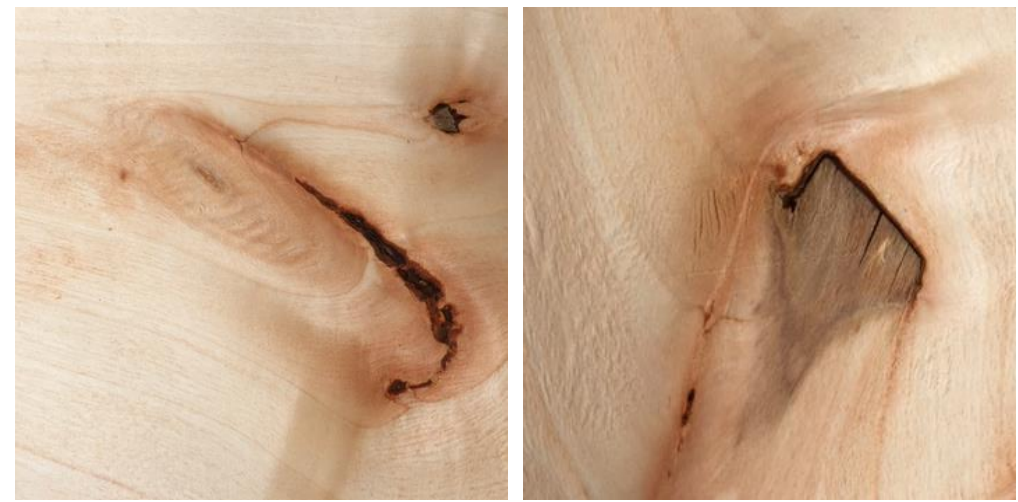

Figura $N^{\circ} 4$ NUDOS NO SANOS, CON INCLUSIÓN DE CORTEZA (IZQ.) Y DECOLORACIÓN (DER.)
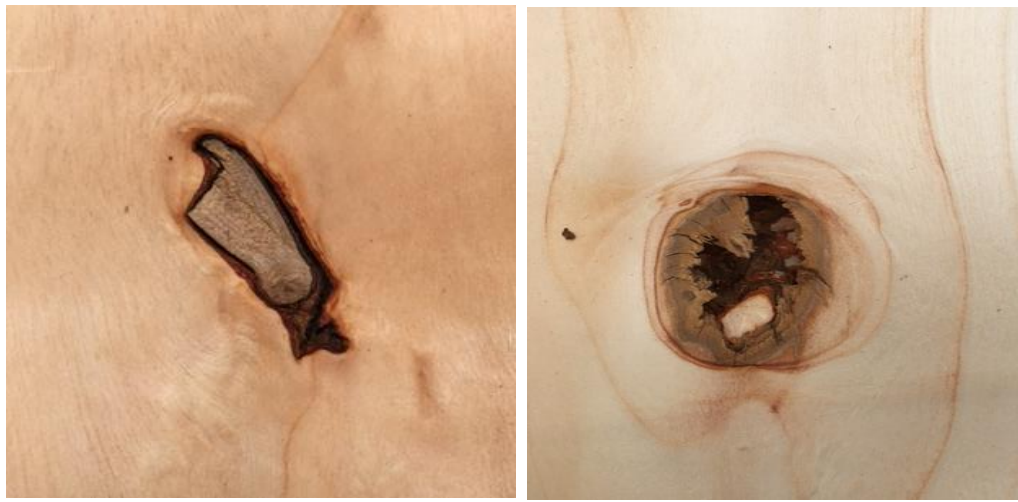

Figura 5

NUDOS MUERTOS 


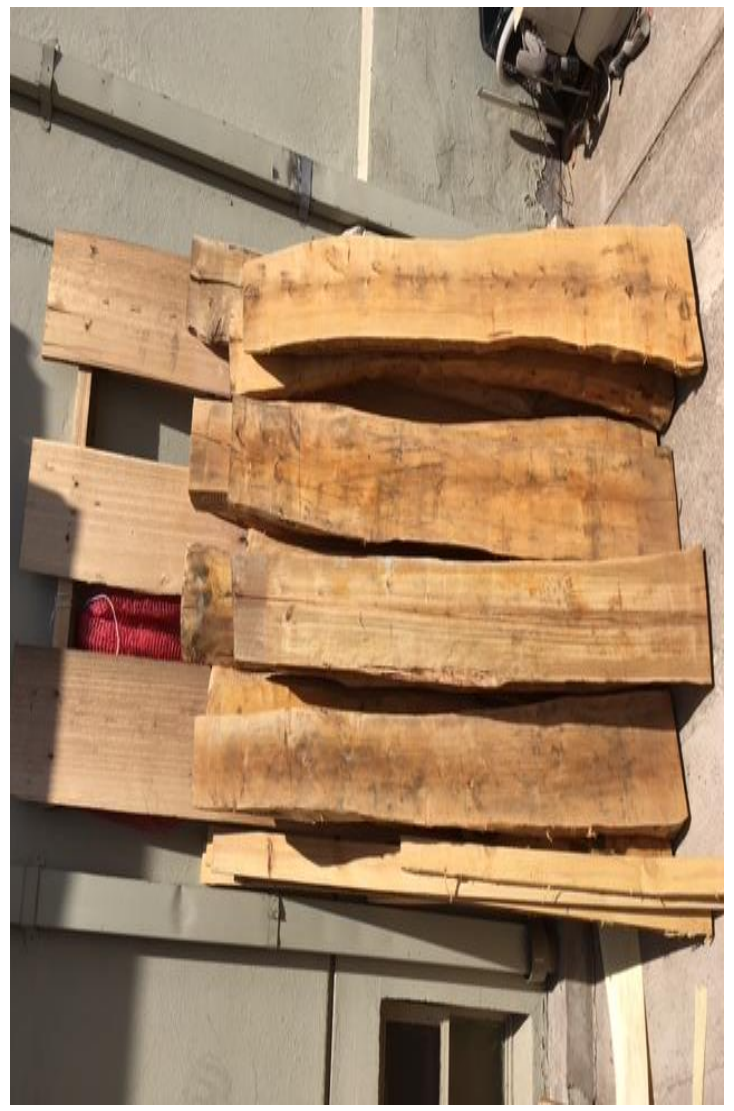

Figura $\mathrm{N}^{\circ} 6$

REMANENTES DE TROZAS DEL PROCESO DE FOLIADO

\section{RESULTADOS}

\section{Rendimientos}

Los rendimientos obtenidos en el proceso de foliado de acuerdo al diámetro y largo de las trozas se presentan en el Cuadro $\mathrm{N}^{\circ} 1$.

La especie que presento un mejor promedio de rendimiento fue el aliso $\left(321,42 \mathrm{~m}^{2} / \mathrm{m}^{3}\right)$, seguido por nogal $\left(260,62 \mathrm{~m}^{2} / \mathrm{m}^{3}\right)$ y por cerezo $\left(140,11 \mathrm{~m}^{2} / \mathrm{m}^{3}\right)$.

Los valores presentan una alta variabilidad en las tres especies ensayadas.

Es importante aclarar que para que sea rentable el negocio de la chapa foliada el rendimiento mínimo debe ser de $350 \mathrm{~m}^{2} / \mathrm{m}^{3}$, independiente del valor de venta, mientras que el porcentaje de residual debería oscilar entre un 20 y $30 \%$. 
Cuadro $\mathrm{N}^{\circ} 1$

CARACTERIZACIÓN DE TROZAS Y RENDIMIENTO EN CHAPA FOLIADA

\begin{tabular}{|c|c|c|c|c|c|c|c|}
\hline \multirow{2}{*}{ Especie } & \multirow{2}{*}{$\begin{array}{l}\text { Diámetro } \\
(\mathrm{cm})\end{array}$} & \multirow{2}{*}{$\begin{array}{l}\text { Largo } \\
(\mathrm{m})\end{array}$} & \multirow{2}{*}{$\begin{array}{c}\text { Volumen } \\
\left(\mathrm{m}^{3}\right)\end{array}$} & \multirow{2}{*}{$\begin{array}{c}\text { Chapa } \\
\left(\mathrm{m}^{2}\right)\end{array}$} & \multicolumn{2}{|c|}{ Volumen Residual } & \multirow{2}{*}{$\begin{array}{c}\text { Rendimiento } \\
\left(\mathrm{m}^{2} / \mathrm{m}^{3}\right)\end{array}$} \\
\hline & & & & & $\left(m^{3}\right)$ & $(\%)$ & \\
\hline Aliso & 22 & 1,6 & 0,077 & 37,2 & 0,018 & 22,73 & 480,37 \\
\hline Aliso & 26 & 1,6 & 0,108 & 20,2 & 0,050 & 46,15 & 186,76 \\
\hline Aliso & 22 & 1,6 & 0,077 & 10,2 & 0,063 & 81,82 & 131,71 \\
\hline Aliso & 22 & 1,6 & 0,077 & 31,2 & 0,028 & 36,36 & 402,89 \\
\hline Aliso & 28 & 1,6 & 0,125 & 62,1 & 0,040 & 32,14 & 495,06 \\
\hline Aliso & 20 & 1,6 & 0,064 & 32,5 & 0,019 & 30,00 & 507,81 \\
\hline Aliso & 22 & 1,6 & 0,077 & 31,2 & 0,011 & 13,64 & 402,89 \\
\hline Aliso & 20 & 1,6 & 0,064 & 26,7 & 0,019 & 30,00 & 417,19 \\
\hline Aliso & 26 & 1,6 & 0,108 & 31,2 & 0,042 & 38,46 & 288,46 \\
\hline Aliso & 30 & 1,6 & 0,144 & 50,1 & 0,058 & 40,00 & 347,92 \\
\hline Aliso & 20 & 1,6 & 0,064 & 21,5 & 0,035 & 55,00 & 335,94 \\
\hline Aliso & 24 & 1,6 & 0,092 & 25,4 & 0,035 & 37,50 & 275,61 \\
\hline Aliso & 26 & 1,6 & 0,108 & 42,6 & 0,021 & 19,23 & 393,86 \\
\hline Aliso & 26 & 1,6 & 0,108 & 27,2 & 0,029 & 26,92 & 251,48 \\
\hline Aliso & 18 & 1,6 & 0,052 & 0,0 & 0,014 & 27,78 & 0,00 \\
\hline Aliso & 22 & 1,6 & 0,077 & 42,3 & 0,014 & 18,18 & 546,23 \\
\hline Aliso & 18 & 1,6 & 0,052 & 0,0 & 0,035 & 66,67 & 0,00 \\
\hline Cerezo & 18 & 1,6 & 0,052 & 0,0 & 0,012 & 22,22 & 0,00 \\
\hline Cerezo & 22 & 1,6 & 0,077 & 21,7 & 0,018 & 22,73 & 280,22 \\
\hline Nogal & 28 & 1,6 & 0,125 & 27,0 & 0,045 & 35,71 & 215,24 \\
\hline Nogal & 22 & 1,7 & 0,082 & 3,4 & 0,049 & 59,09 & 41,32 \\
\hline Nogal & 28 & 1,6 & 0,125 & 31,8 & 0,040 & 32,14 & 253,51 \\
\hline Nogal & 27 & 1,6 & 0,117 & 62,1 & 0,030 & 25,93 & 532,41 \\
\hline
\end{tabular}

\section{Evaluación de Calidad}

Los resultados obtenidos de la aplicación de la clasificación propuesta en este trabajo se presentan en Cuadro $\mathrm{N}^{\circ}$ 2. Cabe señalar que de una troza se obtuvo más de un paquete de chapas.

La calidad observada de las chapas es media a baja, con una preponderancia de las categorías C. para aliso italiano, y B, para aliso negro (dadas sus características, podría servir para elaborar muebles y marcos de cuadros y fotografías), B para cerezo común y nogal común (Figuras $\mathrm{N}^{\circ} 7$ y $\mathrm{N}^{\circ} 8$ ).

No obstante, un $75 \%$ de las chapas producidas no alcanza las dimensiones, en particular el ancho, requerido por el mercado de chapas $(15 \mathrm{~cm}$ mínimo). Esto destaca la no aptitud de este material tan joven para el mercado de chapas, tanto por aspectos dimensionales como cualitativos (Figura $\mathrm{N}^{\circ}$ 9). 
La aplicación de la clasificación empleada por la empresa resultó en que, por las características físicas de las trozas, no se obtienen chapas que alcancen la catalogación de calidad "A" o "AB", ya que, por sus defectos, principalmente nudos, solo se obtuvo calidad "C".

Cuadro $\mathrm{N}^{\circ} 2$

CARACTERIZACIÓN Y CLASIFICACIÓN DE CHAPAS FOLIADAS OBTENIDAS

\begin{tabular}{|c|c|c|c|c|c|c|c|}
\hline \multirow{2}{*}{ Especie } & \multirow{2}{*}{$\begin{array}{c}\text { Troza } \\
\left(\mathbf{N}^{\circ}\right)\end{array}$} & \multirow{2}{*}{$\begin{array}{c}\text { Paquete } \\
\left(\mathbf{N}^{\circ}\right)\end{array}$} & \multirow{2}{*}{$\begin{array}{l}\text { Largo } \\
\text { (cm) }\end{array}$} & \multirow{2}{*}{$\begin{array}{l}\text { Ancho } \\
\text { (cm) }\end{array}$} & \multicolumn{2}{|c|}{ Hojas } & \multirow{2}{*}{ Calidad } \\
\hline & & & & & $\left(\mathrm{N}^{\circ}\right)$ & $\left(\mathrm{m}^{3}\right)$ & \\
\hline Alnus cordata & 16974 & 46 & 140 & 12 & 32 & 5,4 & C \\
\hline Alnus cordata & 16974 & 47 & 140 & 12 & 32 & 5,4 & C \\
\hline Alnus cordata & 16974 & 48 & 140 & 13 & 32 & 5,8 & C \\
\hline Alnus cordata & 16974 & 49 & 150 & 19 & 32 & 9,1 & C \\
\hline Alnus cordata & 17120 & 76 & 140 & 13 & 32 & 5,8 & C \\
\hline Alnus cordata & 17120 & 77 & 140 & 13 & 32 & 5,8 & C \\
\hline Alnus cordata & 20222 & 38 & 155 & 15 & 32 & 7,4 & C \\
\hline Alnus cordata & 20222 & 39 & 150 & 15 & 32 & 7,2 & C \\
\hline Alnus cordata & 20222 & 40 & 155 & 12 & 32 & 6,6 & C \\
\hline Alnus cordata & 20222 & 41 & 155 & 13 & 32 & 6,4 & C \\
\hline Alnus cordata & 20222 & 42 & 110 & 11 & 32 & 3,9 & C \\
\hline Alnus cordata & 20225 & 60 & 180 & 13 & 32 & 7,5 & $\mathrm{C}$ \\
\hline Alnus cordata & 20225 & 61 & 190 & 13 & 32 & 7,9 & C \\
\hline Alnus cordata & 20225 & 62 & 185 & 15 & 32 & 8,9 & C \\
\hline Alnus cordata & 20225 & 64 & 190 & 12 & 32 & 7,3 & C \\
\hline Alnus cordata & 20225 & 65 & 130 & 13 & 20 & 3,4 & C \\
\hline Alnus cordata & 20226 & 66 & 130 & 12 & 32 & 5 & C \\
\hline Alnus cordata & 20226 & 67 & 135 & 12 & 32 & 5,2 & $B$ \\
\hline Alnus cordata & 20226 & 68 & 140 & 15 & 32 & 6,7 & C \\
\hline Alnus cordata & 20226 & 69 & 130 & 13 & 32 & 5,4 & C \\
\hline Alnus cordata & 20226 & 70 & 140 & 10 & 42 & 5,9 & $B$ \\
\hline Alnus cordata & 20229 & 29 & 130 & 13 & 32 & 5,4 & C \\
\hline Alnus cordata & 20229 & 30 & 150 & 12 & 32 & 5,8 & $B$ \\
\hline Alnus cordata & 20229 & 31 & 150 & 13 & 32 & 6,2 & C \\
\hline Alnus cordata & 20229 & 32 & 150 & 14 & 32 & 6,7 & C \\
\hline Alnus cordata & 20229 & 33 & 100 & 14 & 25 & 3,5 & $B$ \\
\hline Alnus cordata & 20229 & 34 & 150 & 15 & 32 & 7,2 & $\mathrm{C}$ \\
\hline
\end{tabular}




\begin{tabular}{|c|c|c|c|c|c|c|c|}
\hline Alnus cordata & 23335 & 35 & 135 & 12 & 32 & 5,2 & $\mathrm{C}$ \\
\hline Alnus cordata & 23335 & 36 & 140 & 13 & 32 & 5,8 & $\mathrm{C}$ \\
\hline Alnus cordata & 23335 & 37 & 120 & 13 & 32 & 5 & C \\
\hline Alnus cordata & 23339 & 57 & 145 & 10 & 32 & 4,6 & $\mathrm{C}$ \\
\hline Alnus cordata & 23571 & 1 & 100 & 11 & 28 & 3,1 & B \\
\hline Alnus cordata & 23571 & 2 & 110 & 10 & 18 & 2 & $\mathrm{C}$ \\
\hline Alnus cordata & 23571 & 3 & 150 & 19 & 29 & 8,3 & $\mathrm{C}$ \\
\hline Alnus cordata & 23571 & 4 & 150 & 13 & 31 & 6 & $\mathrm{C}$ \\
\hline Alnus cordata & 23571 & 5 & 150 & 10 & 41 & 6,2 & $\mathrm{C}$ \\
\hline Alnus cordata & 23571 & 6 & 150 & 12 & 32 & 5,8 & $\mathrm{C}$ \\
\hline Alnus cordata & 23571 & 7 & 140 & 13 & 32 & 5,8 & $\mathrm{C}$ \\
\hline Alnus cordata & 23571 & 8 & 150 & 13 & 32 & 6,2 & C \\
\hline Alnus cordata & 23571 & 9 & 150 & 14 & 32 & 6,7 & $\mathrm{C}$ \\
\hline Alnus cordata & 23571 & 10 & 150 & 13 & 32 & 6,2 & $\mathrm{C}$ \\
\hline Alnus cordata & 23572 & 71 & 210 & 17 & 32 & 11,4 & $\mathrm{C}$ \\
\hline Alnus cordata & 23572 & 72 & 210 & 16 & 32 & 10,8 & C \\
\hline Alnus cordata & 23572 & 73 & 210 & 12 & 35 & 8,8 & $\mathrm{C}$ \\
\hline Alnus cordata & 23572 & 74 & 220 & 18 & 32 & 12,7 & C \\
\hline Alnus cordata & 23574 & 43 & 150 & 13 & 32 & 6,2 & $\mathrm{C}$ \\
\hline Alnus cordata & 23574 & 44 & 155 & 12 & 32 & 6 & $\mathrm{C}$ \\
\hline Alnus cordata & 23574 & 45 & 150 & 12 & 32 & 5,8 & $\mathrm{C}$ \\
\hline Alnus cordata & 23575 & 18 & 140 & 15 & 32 & 6,7 & $\mathrm{C}$ \\
\hline Alnus cordata & 23575 & 19 & 140 & 10 & 30 & 4,2 & B \\
\hline Alnus cordata & 23575 & 20 & 140 & 10 & 32 & 4,5 & B \\
\hline Alnus cordata & 23575 & 21 & 135 & 11 & 32 & 4,8 & $\mathrm{C}$ \\
\hline Alnus cordata & 23575 & 22 & 140 & 12 & 32 & 5,4 & C \\
\hline Alnus cordata & 23575 & 23 & 145 & 13 & 32 & 6 & $\mathrm{C}$ \\
\hline Alnus cordata & 23575 & 24 & 140 & 14 & 30 & 5,9 & $\mathrm{C}$ \\
\hline Alnus cordata & 23576 & 25 & 140 & 12 & 32 & 5,4 & $\mathrm{C}$ \\
\hline Alnus cordata & 23576 & 26 & 140 & 13 & 32 & 5,8 & $\mathrm{C}$ \\
\hline Alnus cordata & 23576 & 27 & 140 & 14 & 32 & 6,3 & B \\
\hline Alnus cordata & 23576 & 28 & 130 & 12 & 32 & 5 & $\mathrm{C}$ \\
\hline Alnus cordata & 23577 & 11 & 150 & 15 & 32 & 7,2 & C \\
\hline Alnus cordata & 23577 & 12 & 150 & 10 & 26 & 3,9 & B \\
\hline Alnus cordata & 23577 & 13 & 150 & 13 & 32 & 6,2 & $\mathrm{C}$ \\
\hline Alnus cordata & 23577 & 14 & 150 & 14 & 32 & 6,7 & $\mathrm{C}$ \\
\hline Alnus cordata & 23577 & 15 & 140 & 12 & 32 & 5,4 & C \\
\hline Alnus cordata & 23577 & 16 & 150 & 17 & 32 & 8,2 & C \\
\hline Alnus cordata & 23577 & 17 & 150 & 17 & 32 & 8,2 & C \\
\hline
\end{tabular}




\begin{tabular}{|c|c|c|c|c|c|c|c|}
\hline Alnus cordata & 23579 & 50 & 150 & 17 & 32 & 8,2 & $\mathrm{C}$ \\
\hline Alnus cordata & 23579 & 51 & 150 & 20 & 32 & 9,6 & C \\
\hline Alnus cordata & 23579 & 52 & 150 & 12 & 32 & 4 & B \\
\hline Alnus cordata & 23579 & 53 & 150 & 20 & 32 & 9,6 & $\mathrm{C}$ \\
\hline Alnus cordata & 23579 & 54 & 150 & 14 & 32 & 6,7 & C \\
\hline Alnus glutinosa & 23332 & 89 & 150 & 12 & 22 & 4 & B \\
\hline Alnus glutinosa & 23332 & 90 & 150 & 15 & 32 & 7,2 & B \\
\hline Alnus glutinosa & 23332 & 91 & 155 & 15 & 32 & 7,4 & A \\
\hline Alnus glutinosa & 23332 & 92 & 145 & 12 & 32 & 5,6 & A \\
\hline Alnus glutinosa & 23332 & 93 & 150 & 13 & 32 & 6,2 & B \\
\hline Alnus glutinosa & 23332 & 94 & 145 & 10 & 32 & 4,6 & B \\
\hline Alnus glutinosa & 23332 & 95 & 145 & 14 & 32 & 6,5 & A \\
\hline Alnus glutinosa & 23332 & 96 & 150 & 14 & 32 & 6,7 & B \\
\hline Alnus glutinosa & 23332 & 97 & 150 & 14 & 32 & 6,7 & B \\
\hline Alnus glutinosa & 23332 & 98 & 130 & 13 & 26 & 4,4 & A \\
\hline Juglans regia & 23344 & 78 & 155 & 15 & 26 & 6 & $B$ \\
\hline Juglans regia & 23344 & 79 & 155 & 12 & 16 & 3 & B \\
\hline Juglans regia & 23344 & 80 & 155 & 12 & 18 & 3,3 & B \\
\hline Juglans regia & 23344 & 81 & 170 & 17 & 32 & 9,2 & B \\
\hline Juglans regia & 23344 & 82 & 135 & 15 & 32 & 6,5 & B \\
\hline Juglans regia & 23344 & 83 & 165 & 13 & 16 & 3,4 & B \\
\hline Juglans regia & 23344 & 84 & 160 & 14 & 18 & 4 & B \\
\hline Juglans regia & 23344 & 85 & 165 & 15 & 32 & 7,9 & $B$ \\
\hline Juglans regia & 23344 & 86 & 155 & 17 & 32 & 8,4 & A \\
\hline Juglans regia & 23344 & 87 & 160 & 14 & 32 & 7,2 & B \\
\hline Juglans regia & 23344 & 88 & 140 & 12 & 30 & 5 & A \\
\hline Prunus avium & 23331 & 99 & 145 & 13 & 32 & 6 & B \\
\hline Prunus avium & 23331 & 100 & 135 & 10 & 38 & 5,1 & $B$ \\
\hline Prunus avium & 23331 & 101 & 140 & 12 & 32 & 5,4 & $\mathrm{C}$ \\
\hline Prunus avium & 23331 & 102 & 135 & 12 & 32 & 5,2 & B \\
\hline
\end{tabular}

Los resultados obtenidos se corresponden con la calidad de las trozas evaluadas, que no son de calidad foliable debido a su forma, diámetro y presencia de nudos vivos.

De hecho, la empresa foliadora normalmente habría rechazado el material, ya que el mercado de la chapa, incluyendo el nacional, requiere chapas homogéneas y sin defectos. 


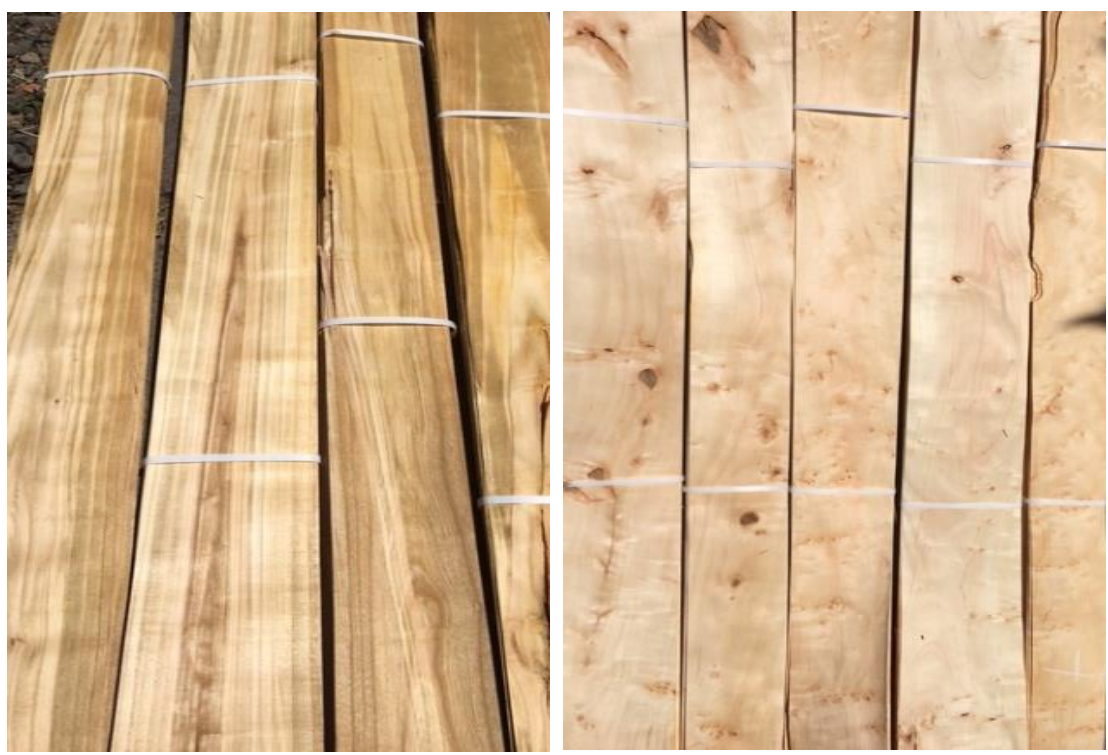

Figura $\mathbf{N}^{\circ} 7$

CHAPAS DE CEREZO COMUUN (IZQ.) Y ALISO ITALIANO (DER.)
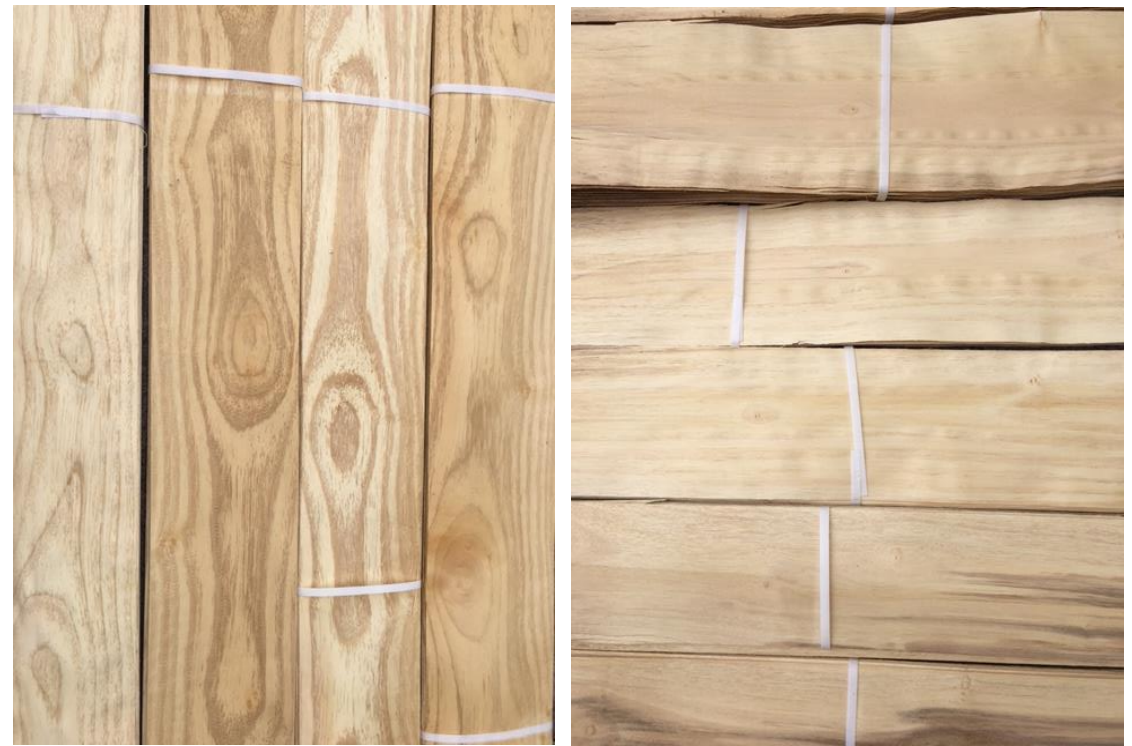

Figura $\mathbf{N}^{\circ} 8$

CHAPAS DE ALISO NEGRO (IZQ.) Y DE NOGAL COMÚN (DER.) 

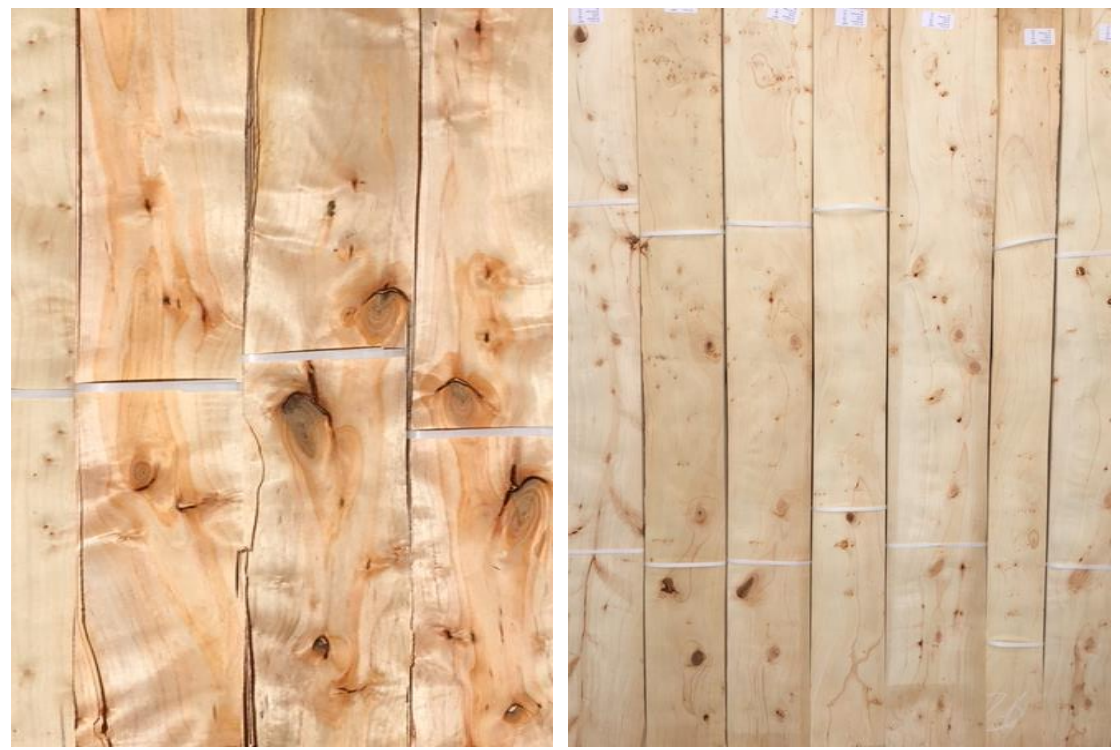

Figura $N^{\circ} 9$

ASPECTO GENERAL DE CHAPAS DE MALA CALIDAD

\section{CONCLUSIONES}

La evaluación de las chapas foliadas realizada a partir de madera de raleo, aplicando la clasificación propuesta, permite concluir que la calidad general de las chapas foliadas es media a baja.

Las mayores limitantes para la obtención de chapas de calidad fueron los pequeños diámetros de las trozas y la presencia de nudos, lo que indica que este material debiera orientarse a otros usos, y enfatiza la necesidad de aplicar un manejo oportuno para usos de mayor valor.

No se observaron defectos asociados a la homogeneidad del color salvo en una troza de aliso italiano. Se obtuvieron chapas tanto de nogal como de aliso en categoría $A$, aunque de anchos que no cumplen con el mínimo para su comercialización.

Si bien con los alisos se obtuvo el mayor rendimiento, no sería una especie comercializable en el mercado tradicional de chapas; es por ello que en este sentido podría realizarse promoción y difusión, ya que presenta características que la haría apta para la elaboración de muebles; particularmente aliso negro podría servir para elaborar también marcos de cuadros y de fotografías.

El foliado de maderas de las especies analizadas, se justificaría con trozas de calidad y de mayores diámetros, las que pueden obtenerse mediante técnicas como la arboricultura de calidad o la silvicultura, maximizando su valor en la comercialización. A ello se suma una tendencia de uso de chapas nudosas, denominadas como rústicas, por lo que desde ese punto de vista podría revalorizarse el producto obtenido en esta evaluación.

Se recomienda continuar realizando evaluaciones de foliado con trozas de mayores diámetros en raleos posteriores o cosecha final, así como evaluar otros usos, como 
chapas rústicas, para material de las características evaluadas en esta oportunidad, a fin de identificar aplicaciones que permitan su valorización.

\section{REFERENCIAS}

Berti, S.; Brunetti, M. y Rescic, L., 2003. Manuale sulla valutazione della qualita degli assortimenti legnosi ritraibili dalle specie legnose pregiate. Regione Lombardia - CNR IVALSA - ERSAF. Milán, Italia. 48 p.

Binkley, D., 2003. Seven decades of stand development in mixed and pure stands of conifers and N-fixing Red Alder. Canadian Journal of Forest Research 33 (2003), pp. 2274-2279.

Borghini, G. y Massafra, M. G., 2002. Legna da Ebanisteria. Ministero per i Beni e le Attivita Culturali. De Luca Editori D'Arte. Roma, Italia, 406 p.

Buresti, E. y Frattegiani, M., 1994. Impianti misti in arboricoltura da legno. Primi risultati in un impianto di farnia (Quercus robur L.) e ontano napoletano (Alnus cordata Loisel). Ann. Ist. Sper. Selv.: 183-199 XXIII.

Ente Nazionale per la Cellulosa e per la Carta, 1990. Principali latifiglie da legno. 80 p.

Gabriel, K.; Blair, I. F. and Mason, W. L., 2005. Growing broadleaves trees on the North York Moors, results after nearly 50 years. Quarterly Journal of Forestry 99:21-30.

Kerr, G. and Evans, J., 1993. Growing broadleaves for timber, Handbook 9, Forestry Commission. 95 p.

Loewe, M. V.; González, O. M. and Balzarini, M., 2013. Wild Cherry tree (Prunus avium L.) growth in pure and mixed plantations in South America. Forest Ecology and Management, pp. 31-41. DOI 10.1016/j.foreco.2013.06.015.

Minotta, G., 2003. L'Arboricoltura da legno: Un'attivita produttiva al servicio dell'ambiente. Ed. Avenue Media. Bologna, Italia, $246 \mathrm{p}$.

Montero, G.; Cisneros, O. y Cañellas, I., 2003. Manual de selvicultura para plantaciones de especies productoras de madera de calidad. Ed. Mundi-Prensa. $284 \mathrm{p}$.

Regione Piemonte, 2001. Guida alla realizzazione e alla gestione degli impianti, Arboricoltura da legno. $111 \mathrm{p}$.

Wiedenbeck, J.; Wiemann. M.; Alderman, D.; Baumgras, J. and Luppold, W., 2004. Defining hardwood veneer log quality attributes. USDA, general Technical Report NE-313. 
